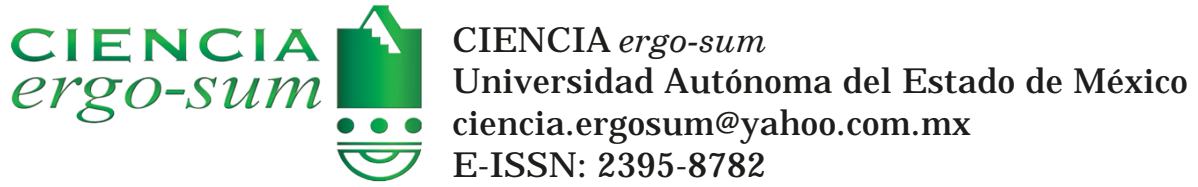

\title{
Indicadores antropométricos y estilos de vida relacionados con el índice aterogénico en población adulta
}

González J aimes, Norma Lilia; Tejeda Alcántara, Adrián Alejandro; Quintín Fernández, E duardo Indicadores antropométricos y estilos de vida relacionados con el índice aterogénico en población adulta CIENCIA ergo-sum, vol. 27, núm. 1, marzo-junio 2020| e74

Universidad Autónoma del Estado de México, México

Esta obra está bajo una Licencia Creative Commons Atribución-NoComercial-SinDerivar 4.0 Internacional .

González J aimes, N. L., Tejeda Alcántara, A. A. y Quintín Fernández, E. (2020). Indicadores antropométricos y estilos de vida relacionados con el índice aterogénico en población adulta. CIENCIA ergo-sum, 27(1). https://doi.org/10.30878/ces.v27n1a6 


\title{
Indicadores antropométricos y estilos de vida relacionados con el índice aterogénico en población adulta
}

Anthropometric indicators and lifestyles related to the atherogenic index in adult population

\author{
Norma Lilia González Jaimes \\ Universidad Autónoma del Estado de Morelos, México \\ psicgonzalez@outlook.com \\ Adrián Alejandro Tejeda Alcántara \\ Universidad Autónoma del Estado de Morelos, México \\ adrian.tejeda@uaem.mx \\ Eduardo Quintín Fernández \\ Universidad Autónoma del Estado de Morelos, México \\ eduardo.quintin@uaem.mx
}

Recepción: 15 de febrero de 2018

Aprobación: 23 de enero de 2019

\section{RESUMEN}

Se determinan los indicadores antropométricos relacionados significativamente con el índice aterogénico (IA) considerando estilos de vida (sedentarismo, promedio de consumo de calorías/día y tabaquismo), edad y sexo en una población adulta, en apariencia sana, adscrita a un hospital de salud pública de México. Método: estudio transversal-observacional. Muestra por conveniencia: $N=206$ (148 mujeres y 58 hombres). Análisis estadísticos: correlacionales y regresión logística binaria utilizando SPSS-19. Resultados y conclusiones: se encuentra una relación significativa entre el incremento de todos los indicadores antropométricos y la elevación del IA independientemente de la edad. Los indicadores cintura/altura y cintura/cadera mostraron en ambos sexos mayor peso en dicha relación.

Palabras ClaVe: indicadores antropométricos, obesidad, índice aterogénico.

\begin{abstract}
The aim of this study was to determine which anthropometric indicators and to what extent are significantly related to the atherogenic index (AI), considering lifestyles (physical inactivity, average calorie consumption/day and smoking), age and sex in an adult population, apparently healthy, attached to a public health hospital in Mexico. Method: Cross-sectional observational study. Sample for convenience: $\mathrm{N}=206$ (148 women and $58 \mathrm{men}$ ). Statistical analysis: Correlational and binary logistic regression using SPSS 19. Results and conclusions: A significant relationship was found between the increase of all anthropometric indicators and the elevation of the AI, regardless of age. The waist/height and waist/hip indicators showed greater weight in this ratio in both sexes.
\end{abstract}

KEYWORDS: Anthropometric indexes, Obesity, Atherogenic index.

\section{INTRODUCCIÓN}

La primera causa de muerte en el mundo se debe a las enfermedades cardiovasculares (OMS, 2014). En 2012 fallecieron 17.5 millones de personas, donde 3 de cada 10 decesos tuvieron origen en dichos padecimientos. Del total mundial de estas pérdidas humanas, $42.29 \%$ correspondió a cardiopatía isquémica y $38.29 \%$ a los accidentes cardiovasculares.

Las estadísticas de defunción en México señalaron a las enfermedades del corazón como la segunda causa de muerte en 2014, las cuales comprendieron cardiopatía isquémica, infarto agudo de miocardio y angina de pecho, tales padecimientos son considerados como manifestaciones clínicas de las afecciones del corazón generadas prin- 
cipalmente por el endurecimiento de las arterias (Aguirre-Botello, 2016). El Instituto Nacional de Estadística y Geografía (INEGI, 2016) reportó 663441 defunciones en la población adulta mexicana en un rango de edad entre 45-60 o más años en el transcurso de 2014, donde los fallecimientos por trastornos cardíacos reflejaron 18.3\%, con un valor absoluto de 121427 muertes (64038 hombres y 57388 mujeres) y por enfermedades isquémicas del corazón que figuraron $12.4 \%$, lo cual comprende 82334 decesos ( 46176 hombres y 36157 mujeres) evidenciando en conjunto $30.7 \%$ del total de pérdidas de vidas humanas, es decir, 3 fallecimientos por enfermedades del corazón por cada 10 decesos anuales. Aunado a estas cifras, INEGI (2016) señaló que las afectaciones cerebro-vasculares representaron 5\% del total de decesos de 2014 con 33166 muertes (16 036 hombres y 17129 mujeres).

El Instituto Nacional del Corazón, los Pulmones y la Sangre (en inglés National Heart, Lung, and Blood Institute. NHLBI) en Estados Unidos definió la aterosclerosis como una enfermedad que se caracteriza por la obstrucción de las arterias causada por el depósito de una placa compuesta por grasas, colesterol, calcio y otras sustancias presentes en la sangre. Los especialistas de dicho instituto explicaron que estas placas, denominadas ateromas, se endurecen con el paso del tiempo y estrechan las arterias limitando el flujo de sangre oxigenada a los órganos y a distintas partes del cuerpo, lo cual aumenta la probabilidad de formación de coágulos potenciando la posibilidad de bloquear la circulación sanguínea de forma parcial o completa, además de generar problemas graves de salud: accidentes cerebro-vasculares o ataques cardíacos que aumentan el riesgo de provocar discapacidad y muerte. El mismo instituto informó que en la actualidad se desconocen las causas específicas de este trastorno, empero, señaló a la falta de actividad física, al hábito tabáquico, a las dietas poco saludables, a la edad y a los aspectos genéticos, entre otros, como agentes de riesgo para el desarrollo de tal padecimiento (NHLBI, 2015). Asimismo, la OMS (2003) declaró que la alimentación inadecuada y el sedentarismo se relacionan con un alto índice de masa corporal, concentraciones altas de lípidos e hipertensión, los cuales son responsables de 75 a 85\% de nuevos casos de cardiopatía coronaria, incluyendo el tabaquismo. En relación con el papel fundamental que tiene la edad en la aterosclerosis, es conveniente señalar que Núñez-García et al. (2007) indicaron que el inicio de dicho trastorno se genera en las dos primeras décadas de vida de los seres humanos y se desarrolla de manera gradual hasta presentar manifestaciones clínicas en edades posteriores. Dichos autores identificaron este padecimiento con un origen multifactorial donde la genética posee un lugar determinante; sin embargo, enfatizaron que los estilos de vida de las personas y la influencia del medioambiente son elementos clave que inciden de modo contundente en su evolución.

En el marco de los estudios preliminares de laboratorio para el diagnóstico del riesgo de aterosclerosis se encuentran los análisis de muestras sanguíneas que determinan el índice aterogénico también denominado índice de Castelli, considerado un potente indicador predictivo de enfermedad coronaria (Scarcella y Després, 2003), el cual se calcula mediante la división del Colesterol Total/Colesterol de Lipoproteínas de Alta densidad (c-HDL) como lo muestra la siguiente fórmula: IA = CT/cHDL (Castelli, 1984) y, de acuerdo con Siniawski et al. (2010), los valores de referencia saludables deben ser menores a $3.25 \mathrm{mg} / \mathrm{dl}$.

Otros factores de riesgo para la aterosclerosis con alta prevalencia en morbi-mortalidad en la población mexicana son el sobrepeso y la obesidad, que la OMS (2015) define como acumulación excesiva de tejido adiposo en el cuerpo que pone en riesgo la salud de quienes los padecen debido a la estrecha relación que han mostrado con diversos padecimientos, por mencionar algunos: las enfermedades cardiovasculares; pulmonares (asma, apnea obstructiva del sueño, síndrome de hipoventilación); del hígado (cirrosis hepática, hígado graso, esteatohepatitis); ginecológicas (anormalidades menstruales, síndrome de ovario poliquístico e infertilidad); cáncer (mama, útero, cérvix, próstata, riñón, esófago, hígado, estómago, páncreas) y síndrome metabólico (Miguel-Soca y Niño-Peña, 2009; OMS, 2014). Con base en la comorbilidad señalada, la Organización para la Cooperación y el Desarrollo Económico (OECD por sus siglas en ingles), identificó el sobrepeso y la obesidad como factores incidentes en la disminución de la esperanza de vida en las personas. Para México específicamente, dicha organización refirió que nuestra nación se ubica en los siete países con menor longevidad, pues alcanza cifras promedio de 74.6 años, mientras que en Japón y España se rebasan los 83 años (OECD, 2015). Tocante a la prevalencia del sobrepeso y la 
obesidad en población adulta mexicana, las encuestas nacionales de salud muestran valores ascendentes durante los últimos años (ENSANUT, 2012). En 2000 la tasa de sobrepeso y obesidad en conjunto fue 61.8\% (hombres 59.5\% y mujeres 64.1\%). En 2006 se elevó a 69.7\% (59.6\% hombres y 72\% mujeres). En 2012 ascendió a 71.2\% (71.2\% hombres y $73 \%$ mujeres) y para 2016 alcanzaron $72.5 \%$, donde $69.4 \%$ correspondió a hombres y $75.6 \%$ a mujeres (ENSANUT MC, 2016).

Referente al tema de la evaluación de la acumulación y distribución de tejido adiposo en el organismo humano, al día de hoy se utilizan diversos indicadores antropométricos; el más utilizado es el índice de masa corporal (IMC) propuesto por Adolph Quetelet en 1835 (Puche, 2005), ratificado más tarde por Keys et al., 1972 y por Garrow y Webster (1985). El IMC es considerado el estándar de oro para la clasificación del infrapeso, normopeso, sobrepeso y los distintos niveles de obesidad (OMS, 2015) y es aplicable a mujeres y hombres adultos entre 20 y 60 años. Para su cálculo, se divide el peso de la persona (en kilos) entre el cuadrado de la estatura (en metros) como lo indica la siguiente fórmula: donde un IMC > a 25 corresponde a sobrepeso, que es identificado como un estado premórbido de la obesidad y un IMC > 30 indica obesidad (OMS, 2015). No obstante, en México, la Secretaría de Salud (2012) en la NOM-043-SSA2-2012 estableció que el punto de corte para la determinación del sobrepeso en la población mexicana en general corresponde a un IMC $>25$ y menor a 30, y un IMC $>23$ y menor a 25 cuando se trata de personas con talla baja, cuyos parámetros se determinan con las siguientes bases: en las mujeres adultas con estatura menor a 1.50 metros y en los hombres con estatura menor a 1.60 metros.

Continuando en la misma línea, en relación con los puntos de corte para el diagnóstico de la obesidad en las personas con talla normal, el IMC debe ser $>30$ y con respecto a la población de talla baja un IMC $>25$. Si bien los puntos de corte para la clasificación del IMC varían de acuerdo con las características étnicas propias de cada región, este índice ha sido blanco de críticas por los especialistas en la materia; entre ellas, se encuentran los aportes realizados por Marfell-Jones et al. (2011), quienes señalan que la determinación del sobrepeso y de la obesidad no discrimina la presencia de la masa grasa y la masa libre de grasa. En consecuencia, indicaron que los atletas o deportistas con una alta masa muscular, a pesar de presentar poca masa grasa, manifestarán un IMC correspondiente a sobrepeso u obesidad. Además, insistieron en la imposibilidad de identificar los sitios corporales donde se concentra la adiposidad mediante el cálculo de dicho índice. En función de estas limitaciones, diversos investigadores han planteado índices alternativos, entre ellos, la evaluación del porcentaje de grasa corporal (\% GC) formulado por Siri (1961) y Durnin y Womersley (1974) que consiste en la medición de cuatro pliegues cutáneos: bíceps, tríceps, subescapular y supraespinal; se determina el porcentaje total de grasa en el cuerpo tomando en consideración el sexo y la edad de los evaluados. Los señalamientos críticos referentes a este tema radican en la falta de discriminación de las zonas corporales de mayor concentración de tejido adiposo, por un lado, y, por el otro, se ha señalado no recomendable en la valoración de individuos obesos debido a que las grandes concentraciones adiposas causan que la lectura de la medida de los pliegues con plicómetro sea inexacta (Marfell-Jones et al., 2011).

Por otra parte, se encuentran los índices antropométricos utilizados para evaluar la adiposidad abdominal o central, los cuales, de acuerdo con diversas investigaciones científicas, se han relacionado significativamente con los síntomas del síndrome metabólico (González-Chávez et al., 2011) y problemas cardiovasculares (Luengo-Pérez et al., 2009). Entre los indicadores de evaluación de la concentración del tejido adiposo en el abdomen más usados se encuentra el índice cintura/cadera (ICC) que, a pesar de utilizarse en múltiples estudios científicos y considerarse como un apropiado indicador y predictor de enfermedades (Gavilan et al., 2002; González-Chávez et al., 2011), se ha señalado que subestima la adiposidad central cuando las personas presentan alto porcentaje de grasa en el cuerpo (un IMC arriba de lo normal) y que a su vez poseen una adecuada proporción corporal obteniéndose un ICC bajo (Koch, 2008). El índice cintura/altura (ICA) también es considerado un adecuado predictor de las enfermedades cardiovasculares (Luengo-Pérez et al., 2009), aunque en contrapartida se ha indicado que carece de puntos de corte para las distintas etnias (Rodríguez y Vélez-Ubiera, 2010). Otro índice usado frecuentemente es la circunferencia de la cintura (CC), cuya crítica se 
centra en no tomar en consideración la estatura (Koch, 2008). Por último, la circunferencia del cuello (Ccu), que tiene la finalidad de valorar la acumulación del tejido adiposo concentrado en la región cervical, también ha mostrado asociación significativa con las enfermedades cardiovasculares (Preis et al., 2010).

En México existen investigaciones enfocadas al estudio de los factores de riesgo cardiovascular y metabólicos en función de los índices somatométricos (Domínguez-Reyes et al. 2017; González-Chávez et al., 2011 y Aguilar-Salinas, 2007). No obstante, los trabajos científicos que establecen una relación entre los indicadores antropométricos y el índice aterogénico son escasos. Entre ellos se encuentran los estudios realizados con niños y adolescentes (Ávila-Curiel et al., 2017 y Arjona-Vicaña et al., 2014) y en población adulta (Saénz-Carrasco et al., 2016).

\section{OвJetivo}

Determinar índices antropométricos y en qué medida se relacionan de manera significativa con el índice aterogénico tomando en consideración estilos de vida (sedentarismo, promedio de consumo diario de calorías y tabaquismo) y factores sociodemográficos (sexo y edad) en una población adulta adscrita a un hospital de salud pública del estado de Morelos, México.

\section{Método}

Se trata de un estudio observacional-transeccional. Participantes: se conformó una muestra no aleatoria y por invitación de $N=206$ (148 mujeres y 58 hombres) adscritos a un hospital de salud pública del estado de Morelos, México, en 2014. La convocatoria para participar en el protocolo de investigación se llevó a cabo mediante carteles ubicados en diversas áreas del nosocomio, pláticas informativas y distribución de trípticos. Los participantes firmaron y entregaron consentimientos informados.

\section{Procedimiento}

El Comité de Bioética del hospital aprobó que se desarrollara el protocolo de investigación. Los participantes leyeron y firmaron sus respectivos consentimientos informados y posteriormente asistieron, previa cita, al laboratorio sede del estudio con ayuno de 12 horas, donde personal especializado, del escenario de este estudio, recolectó las muestras sanguíneas y realizó los análisis pertinentes en relación con la determinación del perfil de lípidos y, a su vez, el cálculo del índice aterogénico. Acto seguido, los participantes acudieron a la evaluación antropométrica que realizaron antropometristas certificados utilizando los criterios de la Sociedad Internacional para el Avance de la Kineantropometría (ISAK por sus siglas en inglés). Para la evaluación antropométrica, se empleó una báscula de bioimpedancia marca Tanita BC 601-F con precisión de 100 gr; para esto, se siguieron las recomendaciones del fabricante con el fin de obtener lecturas exactas: portar poca ropa, pies descalzos y en ayunas. Se midió la estatura con un estadímetro profesional marca Seca 214 con precisión de $1 \mathrm{~mm}$. Se evaluaron los pliegues cutáneos con un plicómetro marca Slim Guide. Para la evaluación de las circunferencias se utilizó una cinta marca Lufkin W606PM graduada en milímetros. Por último, se aplicó un cuestionario para recolectar datos sociodemográficos tales como sexo y edad, así como para la identificación los estilos de vida de los participantes; específicamente, para los niveles de actividad física, se recolectó información del tiempo dedicado y el tipo de actividad tomando como base la clasificación propuesta por el comité de expertos de FAO, WHO y UNU (2004) que define la actividad física como mínima o sedentaria cuando esta práctica no rebasa cinco horas a la semana, en tanto que la actividad física moderada consiste en realizar actividad física entre cinco y menos de diez horas a la semana y, por último, la actividad física vigorosa que consiste en realizar tal actividad más de diez horas a la semana. El consumo 
de calorías promedio/día se obtuvo de los datos arrojados por la báscula de bioimpedancia, cuyo objetivo radica en evaluar la composición corporal.

a) El IMC se obtuvo al dividir el peso en kilogramos entre el cuadrado de la altura en metros utilizando la fórmula propuesta por la OMS (2015). Los puntos de corte utilizados fueron los propuestos por la NOM-043-SSA2-2012 (Secretaría de Salud, 2012).

b) Índice cintura/cadera (ICC): el objetivo consiste en evaluar la cantidad de grasa abdominal. Se utilizó una cinta métrica profesional metálica marca Lufkin W606PM graduada en milímetros. Se midió la circunferencia de la cintura posterior a la inhalación y expiración de aire, en el punto más estrecho, entre el borde inferior de la décima costilla y el borde superior de la cresta ilíaca; el participante estuvo de pie sobre un banco antropométrico, relajado y con los brazos cruzados sobre el tórax. Posteriormente, se midió el diámetro de la cadera ubicando la cinta métrica en el nivel de la protuberancia mayor glútea, perpendicular al eje longitudinal del tronco. El participante estuvo de pie, relajado, con los brazos cruzados sobre el tórax, con los pies juntos y los músculos glúteos relajados.

Se tomaron las medidas por duplicado y se registraron (al detectar diferencias menores a 1\%, se registró el promedio; al existir una diferencia mayor a 1\%, se tomó una tercera medición) y se calculó el índice mediante la fórmula: $\mathrm{ICC}=$ cintura (en centímetros)/cadera (en centímetros). Puntos de corte: mujeres $\leq 0.85$ y hombres $\leq 0.90$ (Barquera et al., 2003).

c) Circunferencia cintura (CC): con la finalidad de valorar la acumulación de grasa abdominal se midió la circunferencia de la cintura en centímetros con el procedimiento descrito en el inciso b. Puntos de corte: mujeres $\leq 80 \mathrm{~cm}$ y hombres $\leq 90 \mathrm{~cm}$ (Secretaría de Salud, 2012).

d) Índice cintura/altura (ICA): con el objetivo de valorar la distribución de la grasa abdominal se evaluó la estatura y la circunferencia de la cintura mediante el procedimiento descrito, y se realizó el cálculo de las medidas obtenidas. Puntos de corte $\leq 0.50$ (Luengo-Pérez et al., 2009).

e) Para obtener el porcentaje de grasa corporal (\%GC) se tomaron medidas de los pliegues cutáneos con un plicómetro marca Slim Guide con precisión de $0.5 \mathrm{~mm}$. Las evaluaciones se realizaron por duplicado y, cuando se presentó diferencia entre las dos medidas por más de $5 \%$, se tomó una tercera medida; de lo contrario, se utilizó el promedio de las dos primeras mediciones: bíceps, tríceps, subescapular y supraespinal. El participante estuvo de pie sobre el piso, con la piel desnuda de los brazos, espalda y abdomen. Para la medición precisa, primero se marcaron los puntos en el cuerpo y se midieron los pliegues de bíceps, tríceps, subescapular y cresta ilíaca con un plicómetro calibrado que ejerció una presión de $10 \mathrm{grs} / \mathrm{mm}^{2}$ sobre el pliegue cutáneo (definido como el espesor de un doble pliegue de piel y tejido adiposo subcutáneo comprimido). Se calculó el porcentaje de grasa corporal mediante las fórmulas propuestas por Durning y Womersley (1974): primero se determinó densidad corporal y posteriormente se calculó el porcentaje de grasa corporal con la fórmula propuesta por Siri (1961) con las siguientes fórmulas: densidad corporal $=\mathrm{C}-\left[\mathrm{M}^{*} \log\right.$ (suma pliegues)], donde $\mathrm{C}$ y $\mathrm{M}$ son constantes respecto a sexo y edad respectivamente; para finalizar, se calculó el porcentaje de grasa Corporal = $\left[(4.95 /(\mathrm{DC}-4.5)]^{*} 100\right.$.

f) Circunferencia del cuello $(\mathrm{CCu})$ : con el propósito de determinar la circunferencia del área cervical se utilizó una cinta métrica profesional metálica marca Lufkin W606PM graduada en milímetros. La cinta se ubicó por encima del cartílago tiroide, perpendicular al eje longitudinal del cuello. La posición de la persona evaluada consistió en estar sentada sobre el banco antropométrico con la cabeza en plano de Frankfurt ya descrito. Aún no se cuentan con puntos de corte por etnia. 


\section{Análisis de datos}

Se llevaron a cabo análisis estadísticos descriptivos para cada una de las variables en estudio. Se utilizó la prueba U de Mann Whitney para la comparación de grupos utilizando variables no paramétricas y para el análisis de variables paramétricas se realizaron correlaciones parciales controlando edad y regresión logística binaria por medio del programa computacional SPSS-19.

\section{Resultados}

\section{CUADRO 1}

Estadísticos descriptivos. Índice aterogénico, indicadores antropométricos y estilos de vida por sexo

\begin{tabular}{|c|c|c|c|c|c|c|c|c|}
\hline & \multicolumn{4}{|c|}{ Mujeres $N=148$} & \multicolumn{4}{|c|}{ Hombres $N=\mathbf{5 8}$} \\
\hline Variables & $\dot{\mathbf{X}}$ & $\sigma$ & $\begin{array}{c}\text { Valores } \\
\text { referencia }\end{array}$ & $\begin{array}{c}\text { Porcentaje }>\text { de } \\
\text { lo recomendado } \\
\text { saludable }\end{array}$ & $\dot{\mathbf{X}}$ & $\boldsymbol{\sigma}$ & $\begin{array}{c}\text { Valores } \\
\text { referencia }\end{array}$ & $\begin{array}{c}\text { Porcentaje }>\text { de } \\
\text { lo recomendado } \\
\text { saludable }\end{array}$ \\
\hline $\begin{array}{l}\text { Índice } \\
\text { aterogénico }\end{array}$ & 3.97 & 1.170 & $<3.25$ & 61.2 & 4.31 & 1.25 & $<3.25$ & 77.6 \\
\hline Peso & 69.87 & 15.05 & - & - & 79.31 & 20.98 & - & - \\
\hline Estatura & 156.93 & 6.39 & - & - & 169.26 & 8.91 & - & - \\
\hline IMC & 28.38 & 5.74 & $\mathrm{IMC}<25$ & $\begin{array}{c}19.2 \text { sobrepeso } \\
+55.5 \text { obesidad } \\
=74.7 \%\end{array}$ & 27 & 5.53 & $\begin{array}{c}\mathrm{IMC}<25 \\
\mathrm{~kg} / \mathrm{m} 2\end{array}$ & $\begin{array}{c}52.8 \text { sobrepeso }+ \\
22.6 \text { obesidad } \\
=75.4 \%\end{array}$ \\
\hline $\mathrm{CC}$ & 86.37 & 13.39 & $80 \mathrm{~cm}$ & 69.9 & 94.47 & 13.46 & $<90 \mathrm{~cm}$ & 53.7 \\
\hline ICC & 0.82 & 0.065 & $<0.85$ & 35.6 & 0.94 & 0.063 & $<0.90$ & 83.3 \\
\hline ICA & 0.55 & 0.088 & $<0.50$ & 73.3 & 0.55 & 0.075 & $<0.50$ & 81.5 \\
\hline$\% \mathrm{GC}$ & 36.70 & 4.65 & $20-30 \%$ & 91.1 & 26.97 & 5.049 & $12-20 \%$ & 94.4 \\
\hline GV & 7.44 & 3.46 & $0-12$ & 9.6 & 9.23 & 4.25 & $0-12$ & 24.5 \\
\hline $\mathrm{Ccu}$ & 35.63 & 3.28 & - & - & 41.23 & 3.34 & - & - \\
\hline $\begin{array}{l}\text { Consumo } \\
\text { Calorías/día }\end{array}$ & 2078.5 & 255.64 & $1600-1800$ & $\begin{array}{c}\text { Consumo alto } \\
\text { calorías/día: } \\
89.7\end{array}$ & 2751.9 & 356.21 & $1800-2000$ & $\begin{array}{c}\text { Consumo Alto } \\
\text { calorías/día: } \\
98.1\end{array}$ \\
\hline $\begin{array}{l}\text { Actividad } \\
\text { física }\end{array}$ & & & & $\begin{array}{c}\text { Sedentarismo: } \\
89.8\end{array}$ & & & & $\begin{array}{c}\text { Sedentarismo: } \\
65.5\end{array}$ \\
\hline $\begin{array}{l}\text { Hábito } \\
\text { tabáquico }\end{array}$ & & $13 \%$ & las participa & intes & & & de los particip & pantes \\
\hline
\end{tabular}

Fuente: elaboración propia.

Nota: $\mathrm{IMC}=$ indice de masa corporal, $\mathrm{CC}=$ circunferencia cintura, $\mathrm{ICC}=$ indice cintura/cadera, ICA $=$ indice cintura/altura, $\% \mathrm{GV}=$ porcentaje de grasa corporal, $\mathrm{GV}=$ grasa visceral, $\mathrm{Ccu}=$ circunferencia del cuello.

La muestra no aleatoria y por invitación se conformó por $N=206$ participantes (148 mujeres y 58 hombres) con un rango de edad de 18-77 años, $\dot{X}=42.96$ años y $\sigma=11.80$. Del total de la población en estudio $(77.6 \%$ hombres y $61.2 \%$ mujeres), $66 \%$ presentó valores de índice aterogénico por arriba de lo saludable recomendado $(3.25 \mathrm{mg} / \mathrm{dl})$ (Siniawski et al., 2010). Asimismo, se observó alta prevalencia en sedentarismo, en conjunto mujeres y hombres (77.65\%); alto promedio de consumo de calorías/día (93.9\%), altos valores de índices antropométricos e índice aterogénico superiores a lo recomendado para la conservación de la salud (cuadro 1). El hábito tabáquico presentó una prevalencia de $18.5 \%$ en conjunto hombres y mujeres. En función de los valores del índice aterogénico, el tabaquismo en mujeres fue de 13\% (6.2\% con índice aterogénico normal y 6.9\% con 
índice aterogénico arriba de lo normal) y en hombres correspondió a 24\% (1.9\% con índice aterogénico normal y $22.2 \%$ con índice aterogénico arriba de lo normal). Mediante los análisis estadísticos U de Mann Whitney, no se encontraron diferencias significativas $(p=0.91)$ entre los grupos de hombres fumadores y no fumadores con los niveles de índice aterogénico.

Por otro lado, los análisis de correlación bivariada entre los indicadores antropométricos, índice aterogénico y el promedio de consumo de calorías/día, controlando por edad, reflejaron en el caso de las mujeres que en la medida que se elevan los indicadores de obesidad hay un incremento en el índice aterogénico, especialmente en Ccu $(r=$ $0.269, p=0.01)$, seguido del ICA $(r=0.267, p=0.01)$, IMC $(r=0.260, p=0.01)$, GC $(r=0.233, p=0.01)$, CC $(r=0.226, p=0.01)$, ICC $(r=0.205, p=0.05)$ y por último el $\% \mathrm{GC}(r=0.198, p=0.05)$, independientemente de la edad. La elevación del promedio de consumo de calorías/día mostró una ligera asociación significativa con el índice aterogénico $(r=0.163, p=0.05)$. Asimismo, se observó que en la medida que se incrementa el promedio de consumo de calorías/día existe una elevación de todos y cada uno de los indicadores de obesidad. Las mayores asociaciones se encontraron con el IMC, CC, Ccu y GV como se muestra en el cuadro 2.

\section{CUADRO 2}

Correlación parcial de indicadores antropométricos, índice aterogénico y promedio consumo calorías/ día por sexo y controlando edad

\begin{tabular}{|c|c|c|c|c|c|c|c|c|c|}
\hline $\begin{array}{l}\text { Variables de control } \\
\text { Edad }\end{array}$ & Índice aterogénico & IMC & $\mathrm{CC}$ & ICC & ICA & $\% G C$ & Ccu & GV & Calorías \\
\hline \multicolumn{10}{|c|}{ Mujeres $(N=148)$} \\
\hline Índice aterogénico & 1.000 & & & & & & & & \\
\hline IMC & $0.260^{* *}$ & 1.000 & & & & & & & \\
\hline $\mathrm{CC}$ & $0.226^{* *}$ & $0.925^{* *}$ & 1.000 & & & & & & \\
\hline ICC & $0.205^{*}$ & $0.449^{* * *}$ & $0.652^{k *}$ & 1.000 & & & & & \\
\hline ICA & $0.267^{* *}$ & $0.919^{* *}$ & $0.963^{* *}$ & $0.684^{* *}$ & 1.000 & & & & \\
\hline$\% G C$ & $0.198^{*}$ & $\mathbf{0 . 7 0 8}^{* *}$ & $0.702^{* *}$ & $0.424^{* *}$ & $0.683^{* *}$ & 1.000 & & & \\
\hline Ccu & $0.269^{* *}$ & $0.824^{* * *}$ & $0.818^{* *}$ & $0.467^{\text {** }}$ & $0.785^{* *}$ & $0.691^{* *}$ & 1.000 & & \\
\hline Grasa visceral & $0.233^{* *}$ & $0.943^{\text {** }}$ & $0.878^{* *}$ & $0.402^{* *}$ & $0.869^{* *}$ & $0.705^{* *}$ & $0.774^{* *}$ & 1.000 & \\
\hline Calorías & $0.163^{*}$ & $0.825^{* *}$ & $0.801^{* *}$ & $0.315^{* *}$ & $0.674^{* *}$ & 0.586 ** & $0.747^{\text {** }}$ & $\mathbf{0 . 7 3 3}^{\text {** }}$ & 1.000 \\
\hline \multicolumn{10}{|c|}{ Hombres $(N=58)$} \\
\hline IMC & $0.347^{* *}$ & 1.000 & & & & & & & \\
\hline $\mathrm{CC}$ & $0.314^{*}$ & $0.917^{\text {*k* }}$ & 1.000 & & & & & & \\
\hline ICC & $\mathbf{0 . 3 0 1}^{*}$ & $0.593^{* * *}$ & $0.775^{\text {** }}$ & 1.000 & & & & & \\
\hline ICA & $0.334^{*}$ & $0.933^{* * *}$ & $0.950^{* *}$ & $0.792^{* *}$ & 1.000 & & & & \\
\hline$\% G C$ & 0.260 & $0.745^{* *}$ & $0.770^{* *}$ & $0.597^{* *}$ & $0.771^{* *}$ & 1.000 & & & \\
\hline Ccu & $0.413^{* *}$ & $0.782^{* *}$ & $0.802^{* *}$ & $0.655^{\text {** }}$ & $0.781^{* *}$ & $0.693^{* *}$ & 1.000 & & \\
\hline GV & $0.299^{*}$ & $0.654^{* *}$ & $0.694^{* *}$ & $0.483^{\text {** }}$ & $0.629^{* *}$ & $0.5966^{* *}$ & $0.665^{* *}$ & 1.000 & \\
\hline Calorías & 0.146 & $0.514^{* *}$ & $0.482^{* *}$ & 0.128 & $0.345^{* *}$ & $\mathbf{0 . 2 9 5}^{*}$ & $0.477^{* *}$ & $0.531^{\text {** }}$ & 1.000 \\
\hline
\end{tabular}

Fuente: elaboración propia.

Nota: ${ }^{* *}$ La correlación es significativa al nivel $0.01 .{ }^{*}$ La correlación es significativa al nivel 0.05 .

$\mathrm{IMC}=$ índice de masa corporal, $\mathrm{CC}=$ circunferencia cintura, $\mathrm{ICC}=$ índice cintura/cadera, ICA = índice cintura/altura, \%GC = porcentaje de grasa corporal, $\mathrm{GV}=$ grasa visceral, $\mathrm{Ccu}=$ circunferencia del cuello.

En el caso de los hombres se elevan los valores de índice aterogénico, independientemente de la edad, en la medida que se incrementan los indicadores de obesidad. Se observó una mayor asociación del índice aterogénico elevado con Ccu $(r=413, p=0.01)$, seguido de IMC $(r=413, p=0.01)$, ICA $(r=334, p=0.05)$, CC $(r=314$, 
$p=0.05)$, ICC $(r=301, p=0.05)$ y por último GV $(r=299, p=0.05)$. El \%GC no mostró asociación significativa. La elevación del promedio de consumo de calorías/día no mostró asociación significativa con el índice aterogénico. No obstante, el promedio de consumo de calorías/día se asoció positivamente de manera significativa con los índices antropométricos de forma descendente con GV, IMC, CC, Ccu, ICA y \% GC (cuadro 2).

De igual manera, se llevaron a cabo análisis multivariados de regresión logística binaria para cada sexo con la finalidad de determinar la existencia de relación significativa y el peso que cada indicador presenta en la relación con el índice aterogénico; se ajustó el modelo por edad y el promedio de consumo de calorías/día (evaluado por bio-impedancia), cuyos resultados se muestran en el cuadro 3 donde se observa que los índices de obesidad con mayor peso en la relación significativa con el índice aterogénico en el caso de las mujeres son ICA $(\beta=4.013, p$ $=0.05)$, lo cual indica que en la medida que aumenta el ICA en una unidad se incrementa cuatro veces el riesgo de elevación del índice aterogénico. En el caso del ICC $(\beta=2.970, p=0.02)$, por cada unidad de aumento en el ICC, el riesgo de elevarse el índice aterogénico es prácticamente tres veces. En torno al incremento en una unidad de $\mathrm{GV}(\beta=1.292, p=0.00)$ el riesgo de elevación del índice aterogénico es de 1.3 veces. En relación con la $\mathrm{Ccu}(\beta=1.219, p=0.03)$ e IMC $(\beta=1.214, p=0.00)$ el incremento en una unidad de éstos eleva el riesgo de aumento en el índice aterogénico en 1.2 veces. Se observó una mínima relación de la CC $(\beta=1.081, p=0.00)$ con el incremento en el riesgo de elevación del índice aterogénico.

En el caso de los hombres, se observó que por el incremento en una unidad del ICC $(\beta=10, p=0.00)$, ICA $(\beta$ $=7.8, p=0.00), \mathrm{Ccu}(\beta=2.167, p=0.00), \operatorname{IMC}(\beta=2.116, p=0.00)$ y GV $(\beta=1.576, p=0.01)$ la posibilidad de elevación del índice aterogénico es de 10 veces: 7.8, 2.2, 2.1 y 1.6 respectivamente. Se observó una mínima relación con $\mathrm{CC}(\beta=1.185, p=0.00)$.

Se señala que la edad y el promedio del consumo de calorías/día no mostraron relación significativa en los análisis de regresión logística binaria en ambos sexos.

\section{CUADRO 3}

Regresión logística binaria. Indicadores antropométricos relacionados con el índice aterogénico por sexo, ajustado por edad y promedio de consumo de calorías

\begin{tabular}{|c|c|c|c|c|c|c|c|c|}
\hline \multicolumn{5}{|c|}{$N=148$ mujeres } & \multicolumn{4}{|c|}{$N=58$ hombres } \\
\hline \multirow{2}{*}{$\begin{array}{l}\text { Indicador antropométrico / } \\
\text { índice aterogénico }\end{array}$} & \multirow[b]{2}{*}{$\operatorname{Exp}(\beta)$} & \multicolumn{2}{|c|}{ I.C. $95 \%$} & \multirow[b]{2}{*}{$\mathbf{p}$} & \multicolumn{3}{|c|}{ I.C. $95 \%$} & \multirow[b]{2}{*}{$\mathbf{P}$} \\
\hline & & $\begin{array}{l}\text { Límite } \\
\text { inferior }\end{array}$ & $\begin{array}{l}\text { Límite } \\
\text { superior }\end{array}$ & & $\operatorname{Exp}(\beta)$ & $\begin{array}{l}\text { Límite } \\
\text { inferior }\end{array}$ & $\begin{array}{l}\text { Límite } \\
\text { superior }\end{array}$ & \\
\hline IMC & 1.214 & 1.067 & 1.381 & 0.003 & 2.116 & 1.275 & 3.513 & 0.004 \\
\hline $\mathrm{CC}$ & 1.081 & 1.023 & 1.143 & 0.005 & 1.185 & 1.066 & 1.319 & 0.002 \\
\hline GV & 1.292 & 1.065 & 1.566 & 0.009 & 1.576 & 1.115 & 2.229 & 0.010 \\
\hline Ceu & 1.219 & 1.015 & 1.463 & 0.034 & 2.167 & 1.369 & 3.432 & 0.001 \\
\hline ICC & 2.970 & 1.162 & 7.595 & 0.023 & 10.00 & 1.998 & 50.042 & 0.005 \\
\hline ICA & 4.013 & 1.509 & 10.671 & 0.005 & 7.800 & 1.655 & 36.760 & 0.009 \\
\hline$\% G C$ & 1.107 & 0.989 & 1.240 & 0.077 & 1.163 & 1.007 & 1.342 & 0.040 \\
\hline
\end{tabular}

Fuente: elaboración propia.

Nota: $\mathrm{IMC}=$ índice de masa corporal, $\mathrm{CC}=$ circunferencia cintura, ICC = índice cintura/cadera, ICA = índice cintura/altura, $\% \mathrm{GC}=$ porcentaje de grasa corporal, $\mathrm{GV}=$ grasa visceral, $\mathrm{Ccu}=$ circunferencia del cuello.

\section{Discusión}

El objetivo de este artículo consistió en determinar cuáles índices antropométricos y en qué medida se relacionan de manera significativa con el índice aterogénico tomando en consideración estilos de vida (sedentarismo, 
promedio de consumo diario de calorías y tabaquismo) y factores sociodemográficos (sexo y edad) en una población adulta adscrita a un hospital de salud pública del estado de Morelos en México. Los resultados obtenidos mediante los análisis estadísticos de correlación bivariada y de regresión logística binaria mostraron relaciones significativas entre la elevación de todos los indicadores antropométricos (IMC, CC, ICC, ICA, \%GC, GV y $\mathrm{Ccu}$ y el incremento del índice aterogénico en ambos sexos, independientemente de la edad de los participantes. Los indicadores antropométricos con mayor peso en dichas relaciones fueron el ICC y el ICA, es decir, en la medida en que aumenta el tejido adiposo abdominal existe un incremento en los niveles del índice aterogénico. Es importante destacar que el grado de relación en dichas variables son sustancialmente mayores en los hombres que en las mujeres (cuadro 3) concordando con los resultados encontrados en un estudio realizado en el estado de Chihuahua en población mexicana por Saénz-Carrasco et al. (2016).

El estudio que se llevó a cabo encontró altas prevalencias de obesidad tanto periférica como abdominal en ambos sexos (cuadro 1), las cuales por sí mismas son factores de riesgo de morbimortalidad (OECD, 2015; OMS, 2015). Las prevalencias en conjunto de sobrepeso y obesidad determinadas por el IMC encontradas en la población participante reflejaron cifras más altas que las reportadas en ENSANUT MC (2016); esto podría deberse a las características de la selección de la muestra poblacional.

Los resultados mostraron alta prevalencia en valores del índice aterogénico por encima de los sugeridos por Siniawski et al. (2010) para la conservación de la salud tanto en mujeres como en hombres, especialmente en éstos últimos (cuadro 1), que coincide con los resultados obtenidos en los estudios realizados por Posadas-Romero et al. (2017) en una muestra poblacional mexicana. Es importante señalar con base en estos resultados que la población participante presenta altos riesgos de padecer aterosclerosis, lo cual puede comprometer a su salud (Aguirre-Botello, 2016; OMS, 2014).

La variable edad no mostró relaciones significativas con el índice aterogénico, contrario a lo planteado por la literatura científica (NHLBI, 2015; Lahoz y Mostaza, 2007), que puede explicarse en función de que los incrementos en los valores del índice aterogénico reflejan alta prevalencia en la población participante.

Entre los factores de riesgo para el desarrollo de aterosclerosis también se encuentra la falta de actividad física (Torres-Damas, 2016; NHLBI, 2015; OMS, 2003). Al respecto, la población de estudio mostró alta prevalencia en sedentarismo, especialmente en las mujeres (cuadro 1); las cifras encontradas en esta investigación son superiores a las reportadas por el INEGI (2018) mediante la encuesta aplicada en 2017 por el Módulo de práctica deportiva y ejercicio físico (Mopradef). Las diferencias entre los datos recabados en este estudio y los informados en dicha encuesta son superiores en $\mathbf{1 7 . 6 \%}$ en hombres y $29.1 \%$ en mujeres. La variable sedentarismo no mostró asociación significativa en los diferentes análisis estadísticos, lo cual posiblemente se explique en función de su alta prevalencia distribuida en todas las edades, así como en las diferentes clasificaciones de obesidad y en los grupos que presentan índice aterogénico normal y arriba de lo recomendable para el mantenimiento de la salud. Las altas prevalencias identificadas en el rubro de sedentarismo representan alto riesgo de enfermedad para la población en estudio. Desde la perspectiva de Torres-Damas (2016), el sedentarismo es un factor asociado al doble de riesgo de muertes prematuras.

En relación con el tabaquismo, se reporta que no se encontraron asociaciones significativas entre éste y el índice aterogénico, aspecto contrario a lo reportado por la literatura científica (NHLBI, 2015; OMS, 2003). Esta categoría puede explicarse en función de la baja prevalencia de tabaquismo encontrada o por la posibilidad de que los participantes hayan minimizado sus consumos reales; situación común en la recolección de datos mediante encuestas de acuerdo con Chen y Qian (2012), quienes enfatizaron que los datos arrojados por encuestas pueden no ser tan confiables debido a que los participantes se sienten expuestos o comprometidos con sus respuestas.

En la evaluación de la composición corporal por bioimpedancia se observaron altos promedios en el consumo de calorías/día, en ambos sexos, especialmente en el grupo de hombres (cuadro 1). De acuerdo con lo recomendado por la Secretaría de Salud (2010), los rangos de consumo de calorías/día sugeridos para el 
mantenimiento de la salud para las mujeres es de 1600 a 1800 calorías/día y para los hombres es de 1800 a 2 000/día, siempre y cuando el IMC corresponda a normopeso. Los resultados obtenidos reflejan consumos de calorías/día mayores a los recomendados para el mantenimiento de la salud aunados a incrementos sustanciales en el IMC en los participantes, lo cual concuerda con los planteamientos de la OMS (2003, 2015) y de Chiquete y Tolosa (2013). La variable promedio de consumo de calorías/día mostró una relación significativa incipiente con el índice aterogénico en los análisis de regresión logística en el caso de las mujeres y una relación no significativa en los hombres. Sin embargo, en los análisis de correlación bivariada controlando por edad mostró asociación positiva y significativa con todos y cada uno de los indicadores antropométricos analizados en este estudio, excepto para el ICC en el caso de los hombres. En el caso de las mujeres se encontraron asociaciones significativas mucho más fuertes entre dichas variables en comparación con el grupo de los hombres (cuadro 2).

\section{Conclusiones}

Todos los indicadores antropométricos considerados presentaron relación positiva y significativa con el índice aterogénico, es decir, en la medida que se elevan las dimensiones de los índices somatométricos se incrementan los puntajes del índice aterogénico. En el caso de las mujeres, y en orden descendente, la correspondencia fue con el ICA, ICC, GV, Ccu, IMC y CC, en tanto que en los hombres fue ICC, ICA, Ccu, IMC, GV y CC. No obstante, los índices con mayor peso en dicho vínculo en ambos sexos fueron el ICA y el ICC, los cuales indican obesidad central o abdominal. Se observaron altas prevalencias en sedentarismo y alta ingesta de calorías/día que por sí mismas son factores de riesgo para una gran diversidad de enfermedades. En la población diana de esta investigación se observó que el aumento de las cifras del promedio en el consumo calórico/día se relaciona con la elevación de la acumulación del tejido adiposo. Del mismo modo, la intensificación de la obesidad robustece los valores del índice aterogénico, lo cual potencia la posibilidad enfermedad cardio y cerebro-vascular en quienes presentan estas condiciones.

Derivado de los resultados, se resalta la importancia de implementar estrategias orientadas al cuidado de la salud que incluyan programas enfocados a la prevención y control: evaluación de la composición corporal, especialmente utilizando los indicadores de obesidad abdominal, establecer esquemas de alimentación nutritiva, práctica de actividad física y erradicación del hábito tabáquico. Las investigaciones longitudinales en relación con las variables de este estudio podrían aportar mayor conocimiento. Asimismo, el análisis de factores socioeconómicos y culturales no tomados en consideración en este proyecto sumarían elementos para una comprensión integral del problema. Los resultados reportados en esta investigación son aplicables únicamente a la muestra participante.

\section{ANÁlisis Prospectivo}

La antropometría es utilizada en diversas disciplinas científicas como la arquitectura, las artes, el deporte, la ergonomía, etc., con el objetivo de evaluar el cuerpo humano en función de su peso, tamaño, forma, constitución y proporción. En las últimas décadas la evaluación de la composición corporal ha cobrado gran importancia en las ciencias de la salud debido a que los índices antropométricos han mostrado relación con diversos indicadores saludables y de enfermedad convirtiéndolos en herramientas económicas (en tiempo y costo) de fácil aplicación y confiabilidad que pueden ser utilizados como valoraciones complementarias de primer contacto para el diagnóstico oportuno de riesgos de enfermedad coadyuvando así a la promoción de la salud poblacional y prevención de enfermedades crónico-degenerativas. Con base en lo expuesto y en los resultados obtenidos en este estudio, surge la necesidad de continuar explorando los posibles vínculos entre los índices antropométricos y los indicadores de salud-enfermedad. 


\section{Agradecimientos}

Se agradecen los comentarios de los árbitros de la revista que mejoraron sustancialmente el contenido del artículo.

\section{REFERENCIAS}

Aguilar-Salinas, C. A. (2007). Adiposidad abdominal como factor de riesgo para enfermedades crónicas. Salud Pública de México, 49, 311-316. Disponible en http://saludpublica.mx/index.php/spm/article/ view/7530/9966

Aguirre-Botello, M. (2016). México, principales causas de mortalidad 1938-2015. Disponible en http://www. mexicomaxico.org/Voto/MortalidadCausas.htm

Ávila-Curiel, A., Galindo-Gómez, C., Juárez-Martínez, L. y Osorio-Victoria, M. L. (2017). Síndrome metabólico en niños de 6 a 12 años con obesidad, en escuelas públicas de siete municipios del Estado de México. Salud Púbica México, 60, 345-403. Disponible en https://doi.org/10.21149/8470

Arjona-Villicaña, R. D., Herrera-Sánchez, L. F., Sumárraga-Ugalde, C. M. y Alcocer-Gamboa, M. A. (2014). Asociación entre el índice de masa corporal y el perfil de lípidos en niños y adolescentes mexicanos con obesidad: un análisis retrospectivo. Boletín Médico del Hospital Infantil de México, 71(2), 88-94. Disponible en http:// www.scielo.org.mx/scielo.php?pid=S1665-11462014000200005\&script=sci_abstract.

Barquera-F., S., Barquera, S., García, E., González-Villalpando, C., Hernández-A., M., Lonngi, G.,... Velásquez, C. (2003). Obesidad en el adulto (E66). Práctica Médica Efectiva, 5(2). México: Instituto de Salud Pública.

Castelli, W. P. (1984). Epidemiology of coronary heart disease: The framingham study. American Journal of Medicine, 76(2A), 4-12. Disponible en https://www.ncbi.nlm.nih.gov/pubmed/6702862

Chen, Y., \& Qian, L. (2012). Association between lifetime stress and obesity in Canadians. Preventive Medicine Journal, 55(5), 464-7. https://doi.org/10.1016/j.ypmed.2012.08.013. Disponible en http://www.ncbi. nlm.nih.gov/pubmed/22944151

Chiquete, E. y Tolosa, P. (2013). Conceptos tradicionales y emergentes sobre el balance energético. Revista de Endocrinología y Nutrición, 21(2), 59-68. Disponible en http://www.medigraphic.com/pdfs/endoc/er2013/er132b.pdf

Domínguez-Reyes, T., Quiróz-Vargas, I., Salgado-Bernabé, A. B., Salgado-Goytua, L., Muñóz-Valle, J. F. y Parra-Rojas, I. (2017). Las medidas antropométricas como indicadores predictivos de riesgo metabólico en una población mexicana. Nutrición Hospitalaria, 34(1), 96-101. Disponible en http://scielo.isciii.es/pdf/ nh/v34n1/15_original.pdf

Durnin, J. V. G. A., \& Womersley, J. (1974). Body fat assessed from total body density and its estimation from skinfold thickness: Measurements on 481 men and women aged from 16 to 72 Years. British Journal of Nutrition, 32,7797. Disponible en https://www.cambridge.org/core/journals/british-journal-of-nutrition/ article/body-fat-assessed-from-total-body-density-and-its-estimation-from-skinfold-thickness-measurements-on-481-men-and-women-aged-from-16-to-72-years/DAC8BA25856FCEB30E22F60E0AF80D07

ENSANUT. (2012). Obesidad en adultos: los retos de la cuesta abajo. Secretaría de Salud. Instituto Nacional de Salud Pública. Disponible en http://ensanut.insp.mx/doctos/analiticos/ObesidadAdultos.pdf

ENSANUT MC. (2016). Encuesta Nacional de Salud y Nutrición de Medio Camino. Informe final de resultados. Instituto Nacional de Salud Pública. Disponible en http://oment.uanl.mx/cifras-de-sobrepeso-y-obesidad-en-mexico-ensanut-mc-2016/ 
FAO/WHO/UNU. (2004). Human energy requirements. Rome, 17-24 October 2001. Disponible en http:// www.fao.org/3/y5686e/y5686e00.htm.

Garrow, J. S., \& Webster, J. (1985). Quetelet'S index (W/H2) as a measure of fatness. International Journal of Obesity, 8(2),147-153.

Gavilan, V. E., Goitia, J., Irala, G. R., Luzuriaga, M. G., Rodríguez, C., Silvia-Costa, J. A. y Brandan, N. C. (2002). Valoración del índice cintura-cadera y su correlación con el riesgo cardiovascular en un hospital de la ciudad de Corrientes. Cátedra II de Medicina. Facultad de Medicina UNNE. Disponible en http://www.unne. edu.ar/unnevieja/Web/cyt/cyt/2002/03-Medicas/M-057.pdf

González-Chávez, Ureña-Lagunes, J., Lavielle-Saramago, M. P. D., Amancio-Chassin, O., Elizondo-Argueta, S. y Hernández-Hernández, H. (2011). Comparación de índices antropométricos como predictores de riesgo cardiovascular y metabólico en población aparentemente sana. Revista Mexicana de Cardiología, 22(2), 59-67. Disponible en http://www.medigraphic.com/pdfs/cardio/h-2011/h112a.pdf

Instituto Nacional de Estadística y Geografía (INEGI). (2016). Principales causas de mortalidad por residencia habitual, grupos de edad y sexo del fallecido. Disponible en https://www.inegi.org.mx/est/contenidos/ proyectos/registros/vitales/mortalidad/tabulados/ConsultaMortalidad.aspad/.

Instituto Nacional de Estadística y Geografía (INEGI). (26 de enero de 2018). Menos de la mitad de la población realiza en su tiempo libre la práctica de algún deporte o ejercicio físico. Comunicado de prensa 25/18. Disponible en http://www.beta.inegi.org.mx/contenidos/saladeprensa/boletines/2018/mopradef/mopradef2018_01.pdf

Keys, A., Fidanza, F., Karvonen, M. J., Kimura, N., \& Taylor, H. L. (1972). Indices of relative weight and obesity. Journal of Chronic Diseases, 25(6-7), 329-343. https://doi.org/10.1016/0021-9681(72)90027-6

Koch, E. (2008). Razón cintura-estatura: un mejor predictor antropométrico de riesgo cardiovascular y mortalidad en adultos chilenos. Nomograma diagnóstico utilizado en el Proyecto San Francisco. Revista Chilena de Cardiología, 27(1), 3-35. Disponible en http://repositorio.uchile.cl/bitstream/handle/2250/128451/130830_C11_ koch_et_al_rev_chil_cardiol_2008_27\%281\%29_23_35.pdf ?sequence $\% 20=\% 201 \&$ isAllowed $\% 20=\% 20 \mathrm{y}$

Lahoz, C. y Mostaza, J. M. (2007). La aterosclerosis como enfermedad sistémica. Revista Española de Cardiología, 60(2), 184-95.Disponible en http://appswl.elsevier.es/watermark/ctl_servlet?_f =10\&pident_articulo=13099465\&pident_usuario $=0 \&$ pcontactid $=$ \&pident_revista $=25 \&$ ty $=10 \&$ accion $=$ L\&origen $=$ cardio\&web $=$ www . revespcardiol.org\&lan $=$ es\&fichero $=25 \mathrm{v} 60 \mathrm{n} 02 \mathrm{a} 13099465 \mathrm{pdf001}$.pdf\&anuncioPdf $=$ ERROR_publi_pdf

Luengo-Pérez, L. M., Urbano-Gálvez, J. M. y Pérez-Miranda, M. (2009). Validación de índices antropométricos alternativos como marcadores de riesgo cardiovascular. Revista Endocrinología y Nutrición, 56(9),439-46.

Marfell-Jones, M., Stewart, A. y Carter, L. (2011). Estándares internacionales para la evaluación antropométrica. Australia: Sociedad internacional para el avance de la kineantropometría.

Miguel-Soca, P. E. y Niño-Peña, A. (2009). Consecuencias de la obesidad. Acimed, 20(4), 84-92. Disponible en de http://scielo.sld.cu/pdf/aci/v20n4/aci061009.pdf. ISSN 1024-9435.

NHLBI (National Heart, Lung and Blood Institute). (2015). ¿Qué es la Ateroesclerosis?. Disponible en https:// www.nhlbi.nih.gov/health-topics/espanol/aterosclerosis.

Núñez-García, M. V., Ferrer-Arrocha, M., Meneau-Peña, T. X., Cabalé-Vilariño, B., Gómez-Nario, O. y Miguelez-Nodarse, R. (2007). Factores de riesgo aterogénico en la población de 19 a 39 años de 2 consultorios del médico de familia. Revista Cubana de Investigaciones Biomédicas, 26(2). Disponible en http://scielo. sld.cu/scielo.php?script = sci_arttext\&pid = S0864-03002007000200006\&lng = es\&tlng = es.

OECD. (2015). Minimum wages after the crisis: Making them pay. Disponible en http://www.oecd.org/social/ Focus-on-Minimum-Wages-after-the-crisis-2015.pdf. 
OMS. (2015). Obesidad y sobrepeso. Fact sheet 311. Disponible en https://www.who.int/en/news-room/factsheets/detail/obesity-and-overweight.

OMS. (2014). Las 10 causas principales de defunción en el mundo 2000-2012. Disponible en http://www.who. int/mediacentre/factsheets/fs310/es/index2.html.

OMS. (2003). Informe sobre la Salud en el Mundo. Capítulo 6. Epidemias mundiales desatendidas: tres amenazas crecientes. Disponible en https://www.who.int/whr/2003/chapter6/es/index1.html.

Posadas-Romero, C., López-Bautista, F., Rodas-Díaz, M. A., Posadas-Sánchez, R., Kimura-Hayama, E., Juárez-Rojas, J. G.(...), Jorge-Galarza, E. (2017). Prevalencia y extensión de la calcificación arterial coronaria en población mexicana asintomática cardiovascular: estudio genética de la enfermedad atersclerosa. Archivos de Cardiología de México, 87(4), 265-368. Disponible en https://www.elsevier.es/es-revista-archivos-cardiologia-mexico-293-articulo-prevalencia-extension-calcificacion-arterial-coronaria-S1405994016301227.

Puche, R. C. (2005). El índice de masa corporal y los razonamientos de un astrónomo. Medicina (Buenos Aires), 65(4), 361-365. Disponible en http://www.scielo.org.ar/scielo.php?script=sci_arttext\&pid= 0025-76802005000400016\&lng=es\&tlng=es.

Rodríguez, B. y Vélez-Ubiera, R. (2010). Relación entre perfil lipídico e índices de masa corporal en estudiantes universitarios del INTEC. Ciencia y Sociedad, 35, 371-385. Disponible en https://www.redalyc.org/ articulo.oa?id=87020009001.

Preis, S. R., Massaro J. M., Hoffman, U., D’Agostino, R. B. Sr., Levy, D., Robins, S. J., Meigs, J. B., O’Donell, C. J., \& Fox, S. S. (2010). Neck circumference as a novel measure of cardiometabolic risk: The Framingham heart study. Journal of Clinical Endocrinology \& Metabolism, 56(9),439-46. Disponible en https://www. ncbi.nlm.nih.gov/pubmed/20484490.

Saénz-Carrasco, J. A., Muñoz-Daw, M. J., Hinojos-Seáñez, E. y De la Torre-Díaz, M. L. (2016). Riesgo cardiovascular en los empleados de la Universidad Autónoma de Chihuahua, México. Nutrición Clínica y Dietética Hospitalaria, 36(3), 45-52. https://revista.nutricion.org/PDF/saenzcarrasco.pdf.

Scarcella, C. y Després, J. P. (2003). Tratamiento de la obesidad: necesidad de centrar la atención en los pacientes de alto riesgo caracterizados por la obesidad abdominal. Cadernos de Saúde Pública, 19, S7-S19. http:// www.scielo.br/scielo.php?script=sci_arttext\&pid=S0102-311X2003000700002\&lng=es\&tlng=es.

Secretaría de Salud. (2012). Norma Oficial Mexicana NOM-043-SSA2-2012. Para el manejo integral de la Obesidad. Disponible en http://dof.gob.mx/nota_detalle.php?codigo=5285372\&fecha=22/01/2013

Secretaría de Salud. (2010). Guía de Alimentos para la población mexicana. México: Press Printing. Disponible en http://www.imss.gob.mx/sites/all/statics/salud/guia-alimentos.pdf

Siniawski, D. A, Masson, W., Sorroche, P., Casañas, L., Krauss, J., \& Cagide, A. (2010). Correlation between Apolipoprotein B-to-Apolipoprotein A1 Ratio and Total-to-HDL-Cholesterol Ratio in a Healthy Population: Should Castelli Index be Updated? Revista Argentina de Cardiología, 79(1), 33-38. Disponible en http://www.scielo.org.ar/pdf/rac/v79n1/en_v79n1a10.pdf

Siri, W. E. (1961). Body composition from fluid space and density. In J. Brozek, \& A. Hanschel, (Eds.). Techniques for measuring body composition (pp. 223-244). Washington D. C.: National Academy of Science.

Torres-Damas, E. L. (2016). Factores de riesgo aterogénico en población adulta venezolana. Revista Cubana de Angiologia, 17(1), 44-54. Disponible en https://www.medigraphic.com/pdfs/revcubangcirvas/cac-2016/ cac161g.pdf.

CC BY-NC-ND 